\title{
Correction: Pediatric literature trends: high-level analysis using text-mining
}

Sarina Levy-Mendelovich, Yiftach Barbash, Ivan Budnik, Daniella Levy-Erez, Raz Somech, Shelly Soffer, Susan Furth and Eyal Klang

(c) The Author(s), under exclusive licence to the International Pediatric Research Foundation, Inc 2021

Pediatric Research (2021) 90:234; https://doi.org/10.1038/s41390-021-01644-x

Correction to: Pediatric Research https://doi.org/10.1038/s41390021-01415-8, published online 17 March 2021

In this article, the captions for Figs. 1, 3, and 5 were inadvertently reproduced incorrectly. The corrected captions are given below:

\begin{tabular}{r|r} 
Citation/publication ratio & \\
United States & 6.20 \\
United Kingdom & 5.89 \\
Sweden & 5.78 \\
Netherlands & 5.61 \\
Finland & 5.52 \\
Canada & 5.18 \\
Australia & 4.77 \\
Switzerland & 4.47 \\
Germany & 4.16 \\
Italy & 3.63 \\
Taiwan & 3.61 \\
Israel & 3.50
\end{tabular}

Fig. 1 Citations/publications ratio per country. Citations/publications ratio per country over the past 20 years.

\begin{tabular}{r|r} 
Citation/publication ratio & \\
Guidelines & 12.20 \\
Meta-analysis & 10.64 \\
RCT & 6.54 \\
Multicenter study & 6.51 \\
Clinical trial & 6.02 \\
Review & 5.90 \\
Editorial & 2.82 \\
Letter & 1.52
\end{tabular}

Fig. 3 Citations/publications ratio per article type. Citations/ publications ratio per article type over the past 20 years.

\section{Citation/publication ratio}

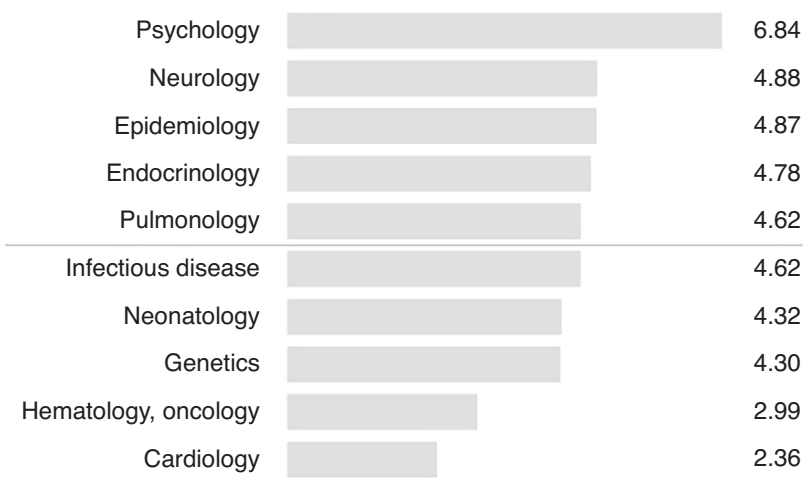

Fig. 5 Citations/publications ratio per topic. Citations/publications ratio per topic over the last 20 years. 